ИЗВЕСТИЯ АКАДЕМИИ НАУК ЭСТОНСКОИ ССР. ФИЗИКА * МАТЕМАТИКА PROCEEDINGS OF THE ACADEMY OF SCIENCES OF THE ESTONIAN SSR. PHYSICS * MATHEMATICS

$1985,34,1$

\title{
ЧИСЛЕННОЕ ИССЛЕДОВАНИЕ ВИХРЕВЫХ КОЛЕЦ
}

\author{
(Представил Н. Эпик)
}

Қлассические результаты для круговой вихревой нити, полученные в рамках теории невязкой жидкости, широко используются в гидродинамике, аэродинамике и метеорологии для описания ряда явлений.

Настоящая работа посвящена развитию этих результатов на базе современных вычислительных средств с целью описания эволюции вихревого кольца в реальной жидкости.

В эксперименте как для ламинарного, так и для турбулентного режима течения наблюдается автомодельное развитие вихревого кольца конвективного происхождения (т.н. термика), обусловленное действием силы Архимеда, определяющей постоянное отношение увеличивающегося радиуса кольца к высоте его подъема в любой момент времени $r_{m} / b\left[{ }^{1}\right]$ (рис. 1 ).

В случае динамического кольца экспериментальные данные говорят о другом $\left[{ }^{2}\right]:$ в ламинарном режиме его радиус растет незначительно, и только при развитой турбулентности радиус кольца начинает меняться резко, и кольцо распадается вследствие неустойчивости. Однако в дальнейшем после фазы неустойчивости почти всегда формируется некоторая устойчивая форма вихря, для которой фиксируется постоянство отношения ее радиуса к высоте подъема. На этом основано обращение к автомодельной постановке задачи для описания турбулентного вихревого кольца $\left[{ }^{3-5}\right]$.

Ниже рассматривается подход к описанию динамического вихревого кольца на основе нестационарных уравнений движения несжимаемой жидкости.

Перенос завихренности $\zeta$ описывается уравнением

$$
\frac{\partial \zeta}{\partial t}+\frac{\partial}{\partial r}(v \zeta)+\frac{\partial}{\partial x}(u \zeta)=v\left[\frac{\partial^{2} \zeta}{\partial x^{2}}+\frac{\partial^{2} \zeta}{\partial r^{2}}+\frac{q}{r} \frac{\partial \zeta}{\partial r}-\frac{q \zeta}{r^{2}}\right]
$$

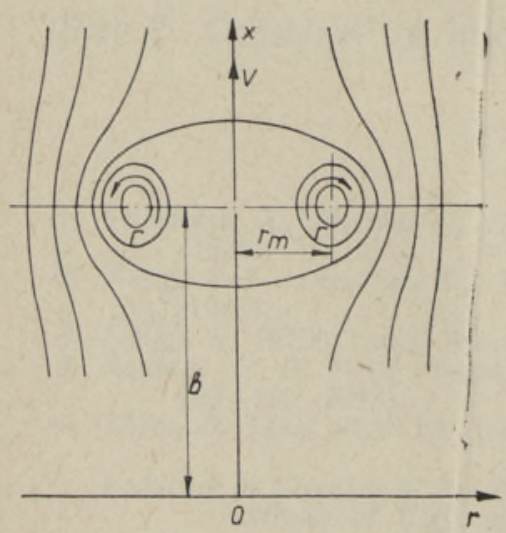

которое при $q=1$ соответствует осесимметричной задаче, а при $q=0$ - плоской; $x, r-$ оси цилиндрической системы координат при $q=1$ или оси декартовой системы при $q=0$, $v$ - коэффициент молекулярной вязкости. Функция тока введена формулами:

$$
u=\frac{1}{r^{q}} \frac{\partial \psi}{\partial r}-V(t), \quad v=-\frac{1}{r^{q}} \frac{\partial \psi}{\partial x},
$$

Рис. 1. Параметры вихревого кольца. 
и может быть найдена как решение уравнения Пуассона

$$
-\zeta r^{q}=\frac{\partial^{2} \psi}{\partial x^{2}}+\frac{\partial^{2} \psi}{\partial r^{2}}-\frac{q}{r} \frac{\partial \psi}{\partial r} .
$$

Здесь $V(t)$ - скорость вихревого кольца, равная $d x_{0}(t) / d t$, где $x_{0}(t)-$ пройденное вихрем расстояние. Начальное условие определяется условиями образования вихревого кольца или вихревой пары: при $t=0$ функция $\zeta(r, x, 0)$ равна нулю всюду, кроме точки $r=a$, где она бесконечна (круговая вихревая нить радиуса $a$ при $q=1$ или две противоположно направленные прямолинейные вихревые нити на расстоянии $2 a$ при $q=0$ ).

Краевые условия

$$
\begin{gathered}
\zeta=\psi=0 \quad \text { при } r=0 \quad \text { (условие симметрии), } \\
\zeta, \psi \rightarrow 0 \text { при } x^{2}+r^{2} \rightarrow 0 .
\end{gathered}
$$

Интегрируя (1) при условиях (3), получим условие сохранения

$$
\left(\frac{2 \pi}{2}\right)^{q} \int_{-\infty}^{\infty} \int_{0}^{\infty} r^{q+1} \zeta(r, x, t) d x d r=B, \quad B=\text { const. }
$$

Последнее выполняется для любого осесимметричного распределения вихря. Решение линеаризованной задачи (1) - (4) приведено в $\left[{ }^{6,7}\right]$.

Соответствующая краевая задача в безразмерных переменных имеет вид:

$$
\begin{gathered}
\frac{\partial \zeta}{\partial t}+\frac{\partial}{\partial r}(v \zeta)+\frac{\partial}{\partial x}(u \zeta)=\frac{1}{R e}\left(\frac{\partial^{2} \zeta}{\partial x^{2}}+\frac{\partial^{2} \zeta}{\partial r^{2}}+\frac{q}{r} \frac{\partial \zeta}{\partial r}-\frac{q \zeta}{r^{2}}\right), \\
\frac{\partial^{2} \psi}{\partial x^{2}}+\frac{\partial^{2} \psi}{\partial r^{2}}-\frac{q}{r} \frac{\partial \psi}{\partial r}=-r^{q} \zeta \\
\frac{\partial C}{\partial t}+v \frac{\partial C}{\partial r}+u \frac{\partial C}{\partial x}=\frac{1}{R e S c}\left(\frac{\partial^{2} C}{\partial x^{2}}+\frac{\partial^{2} C}{\partial r^{2}}+\frac{q}{r} \frac{\partial C}{\partial r}\right), \\
\psi=\zeta=\frac{\partial C}{\partial r}=0, \quad v=-\frac{1}{r^{q}} \frac{\partial \psi}{\partial x}=0, \quad r^{q} u=\frac{\partial \psi}{\partial r}=0, \quad(r=0) \\
\psi=\zeta=C=0 ; \quad\left(x^{2}+r^{2} \rightarrow \infty\right) .
\end{gathered}
$$

Здесь уравнение $(7)$ описывает перенос примеси $(C-$ ее концентрация),

$$
\begin{aligned}
& x_{1}=x / a, \quad r_{1}=r / a ; \quad u_{1}=u / V_{0}, \quad v_{1}=v / V_{0}, \quad \zeta_{1}=\zeta a / V_{0}, \\
& \psi_{1}=\psi / a^{q+1} V_{0}, \quad t_{1}=t\left(V_{0} / a\right)=s / s_{0}, \quad R e=V_{0} a / v \\
& C_{1}=C / C_{0}, \quad S_{C}=v / D
\end{aligned}
$$

$D$ - коэффициент диффузии, индекс 1 ниже опущен.

Решение (5)-(8) проведено неявным методом поперечно-продольных направлений при аппроксимации конвективных членов по Аракаве. В этих целях якобиан

$$
J(\zeta, \psi)=\frac{\partial \psi}{\partial r} \frac{\partial \zeta}{\partial x}-\frac{\partial \psi}{\partial x} \frac{\partial \zeta}{\partial r}
$$

при $q=1$ заменялся на форму $J(\zeta / r, \psi)$.

Граничные условия из бесконечности наносились на границы рас- 
четного прямоугольника. В целях более точного определения значёний $\psi$, необходимых для вычисления скорости вихревого кольца, уравнения (5) и (6) рассчитывались на разных сетках (уравнение Пуассона на сетке $61 \times 61$, а уравнение (5) на сетке $31 \times 41)$. Решение уравнения Пуассона выполнено с постоянным значением итерационного параметра при значениях $\varepsilon=10^{-5}-10^{-4}$ и с дополнительным условием - ограничением числа итераций внутреннего цикла $\left(s_{\max }=91\right)$. Внутренние итерации прекращались при выполнении условия

$$
\frac{\left|\psi_{\max }^{s+1}-\psi_{\max }^{s}\right|}{\psi_{\max }^{s+1}}<\varepsilon .
$$

Начальное условие задавалось в виде аналитического решения для распределения вихря [ $\left.{ }^{6}\right]$.

Скорость вихревого кольца при $q=1$ выражалась через интегральные характеристики течения по формулам [ $\left.{ }^{7,8}\right]$

$$
\begin{gathered}
V(t)=\frac{1}{2 B}[E(t)-F(t)], \\
E(t)=\pi \int_{\infty}^{\infty} \int_{0}^{\infty} \psi \zeta d x d r, \\
F(t)=2 \pi \int_{-\infty}^{\infty} \int_{0}^{\infty}\left(r^{2} u \zeta-x r v \zeta\right) d x d r, \\
B=\pi \int_{-\infty}^{\infty} \int_{0}^{\infty} r^{2} \zeta d x d r,
\end{gathered}
$$

где $E(t)$ - кинетическая энергия и $B$ - постоянный импульс вихревого кольца. Для плоской задачи $(q=0)$

$$
V(t)=\frac{\Gamma(t)}{4 \pi r_{m}}, \quad \Gamma(t)=\int_{-\infty}^{\infty} \int_{0}^{\infty} \zeta d x d r,
$$

где $\Gamma(t)$ - циркуляция вихря, $r_{m}$ - расстояние до $\zeta_{\max }$.

При использовании схемы $(\psi, \zeta)$ функция тока относительного движения определялась следующим образом:

$$
\psi_{\text {отн }}=\psi-\frac{V(t) r^{2}}{?} .
$$

В алгоритме расчетная область сдвигалась за движущимся динамическим элементом с определением скорости вихря на каждом временном слое по формулам (10), (14).

Основные расчеты проводились в пределах изменения чисел $R e=10-1000$ при $B=1$. В случае больших $1 / \sqrt{p}$ можно выписать приближение $\zeta_{0}$ из $\left[{ }^{6}\right]$ в виде $\left(s / s_{0}=1\right)$

$$
\zeta_{0}=\frac{1}{2 \pi^{2} \sqrt{r} p_{1}} e^{-\frac{\left(x-x_{0}(t)\right)^{2}+(r-1)^{2}}{2 p_{1}}},
$$

где $p=2 t / R e, p_{1}=p / t$.

Проведенные расчеты позволяют сделать следующие выводы. Из (15) видно, что при увеличении числа $R e$ начальное распределение завихренности становится более концентрированным. Согласно расчетным данным, его диффузия происходит тем медленнее, чем больше 


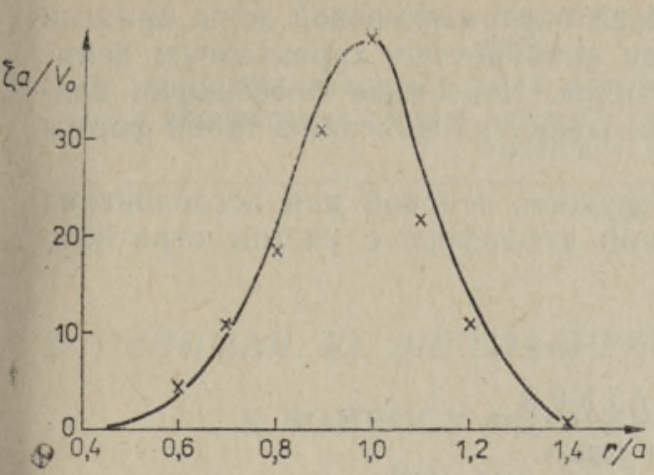

Рис. 2. Сравнение расчетных (-) и экспериментальных (X) значений $\zeta\left(x_{0}(t) r\right)$ при $t=1,4$ (условия расчета соответствуют данным эксперимента).

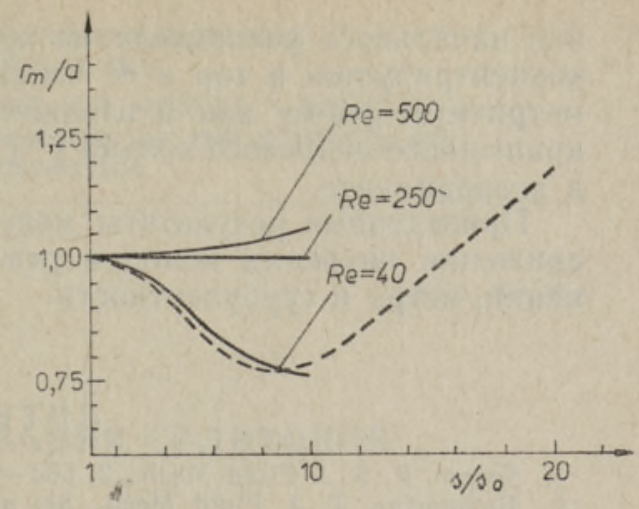

Рис. 3. Изменение радиуса вихревого кольца с течением времени. Сплошные линии - численное решение полной задачи, пунктирная линия - решение линеаризованной задачи.

число Re. Сравнение расчетных данных для завихренности с данными эксперимента при использовании лазерного доплеровского измерителя скорости [ $\left.{ }^{9}\right]$ указывает на их удовлетворительное качественное и количественное согласие для начальной стадии развития кольца (рис. 2). Последнее говорит в пользу выбранной в расчете формы начального распределения вихря. Дальнейшая эволюция вихревого кольца отличается от поведения, описываемого линейным решением [ $\left.{ }^{6}\right]$. В частности, при увеличении $R e(R e \geqslant 350)$ фиксируется рост радиуса вихревого кольца $r_{m}-$ кольцо растягивается (рис. 3 ). При этом отлично от линейного решения вихревое кольцо сохраняет тороидальную форму, а его скорость падает не столь резко (рис. 4). При значениях $R e>100$ в системе координат, связанной с вихрем, наблюдается его деформация. Изолинии завихренности начинают отличаться от симметричных (описываемых решением линеаризованной задачи), несколько вытягиваются в сторону, противоположную направлению движения вихревого кольца и поворачиваются относительно оси симметрии под некоторым углом. Является характерным, что изолинии больших значений вихря деформированы сильней изолиний меньших значений. Дальнейшая эволюция этих асимметричных распределений во времени сопровождается выравниванием значений вихря по всей вихревой зоне вследствие диффузии. При больших временах влияние нелинейности исчезает и должны достигаться предельные формы распределений завихренности и функции тока, описываемые автомодельным решением $\left[{ }^{10}\right]$. Замена вязкости $v$ на зависящую от времени $\left[{ }^{4}\right]$ приводит к режиму, когда влияние нелинейности остается постоянным фактором развития кольца, и его эволюция при $t \rightarrow \infty$ идет по другому закону автомодельности [ $\left.{ }^{4,5}\right]$.

Расчеты переноса пассивной примеси, проведенные после определения полей скорости вихря, показывают, что при зада-

Рис. 4. Изменение скорости вихревого кольца с течением времени.

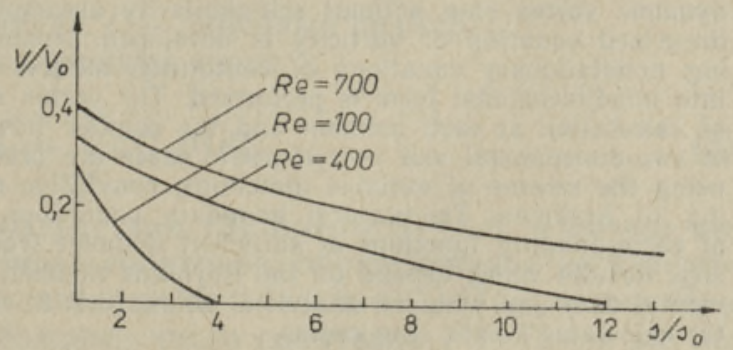


нии начального распределения примеси внутри вихревой зоны примесь концентрируется в тор и ее изолинии приобретают характерную асимметричную форму как и изолинии вихря. Известные фотопрафии подкрашенного вихревого кольца $\left[{ }^{11}\right]$ указывают на фиксацию такой формы в эксперименте.

Приведенные результаты могут служить основой для исследования движения вихревых колец в реальной атмосфере с учетом стратификации, ветра и турбулентности.

\section{Л И Т Е Р А Т Р А}

1. Scorer, R. S. J. Fluid Mech., 2, 583-593 (1957).

2. Maxworthy, T. J. Fluid Mech., 81, part 3, 465-495 (1977).

3. Lilly, D. K. J. Atmos. Sci, 21, 83-98 (1964).

4. Луговцов Б. А. В кн.: Некоторые проблемы математики и механики. М., «Наука», 1970, 182-189.

5. Капланский Ф. Б., Эпитейн А. М. Изв. АН ЭССР. Физ. Матем., 25, № 4, 408417 (1976).

6. Капланский Ф. Б. Изв. АН ЭССР. Физ. Матем., 33, № 3, 372-374 (1984).

7. Калтаев А. В кн.: Динамика сплошной ореды. Алма-Ата, КазГУ, 1982, 63-70.

8. Saffman, P. G. Stud. Appl. Math., 49, 370-380 (1970).

9. Sullivan, J. P., Widnall, S. E., Ezekiels, S. AIAA Journal, 11, № 10, 1384-1389 (1973).

10. Ериин Ш. А., Калтаев А. В кн.: Прикладная механика. Применение математических методов в естествознании. Алма-Ата, КазГУ, 1979, $164-169$.

11. Okabe, J., Inoue, S. Rep. Res Inst. Appl. Mech., Kynshu Univ., 8, 32-91 (1960).

Институт термофизики и электрофизики Академии наук Эстонской ССР
Поступила в редакцию 13/VI 1983

\section{F. KAPLANSKI}

\section{KEERISRŌNGASTE UURING ARVMEETODIL}

On pakutud välja uudne vōimalus keerisrōngaste mittemodelseks matemaatiliseks kirieldamiseks kokkusurumatu vedeliku mittestatsionaarsete liikumisvõrrandite arvmeetodil lahendamise abil, kusjuures keeristsooni kinnihoidmiseks arvutuspiirkonnas on kasutatud nihkeprotseduuri keerise taga ja keerise laienemine on kompenseeritud koordinaadi sammu suurendamisega. Olesande lahend on saadud muutuvate suundade skeemi järgi, kasutades konventiivsete liikmete puhul Arakava aproksimatsiooni.

Uuringu tulemused on vastandatud autori poolt lahendatud automodelsele ülesandele ning võrreldud neid katseandmetega. On analüüsitud varem kasutatud automodelse ülesandepüstituse piiratuse pōhjusi vooluse turbulentse režiimi korral.

\section{F. KAPLANSKI}

\section{NUMERICAL SOLUTION FOR VORTEX RING}

Development of a vortex ring resulting upon release of a discrete mass of fluid into media of the same properties with some initial momentum is considered. The problem with initial condition of special form is formulated. An approach for simulating dynamic vortex ring without self-similarity assumption based on analytical solution of linearized equation of vorticity is developed. Numerical integrating of the corresponding nonstationary equations of continuity, motion and admixture transfer transformed into nondimensional form is performed. The vortex structure is maintained at the sphere of calculation at each moment and the counter flow velocity could be deduced. Results of two-dimensional and axisymmetric cases are presented. Calculations were performed, using the scheme of variable directions, convective members being approximated according to Arakawa. The natural boundary conditions of infinity are replaced by values of corresponding functions at sufficient distance from the position of concentrated vortisity. Results which depend on the Reynolds number, are compared with available experimental data and with the analytical solution. It is established that nonlinearity increases the calculated vortex ring radius. 